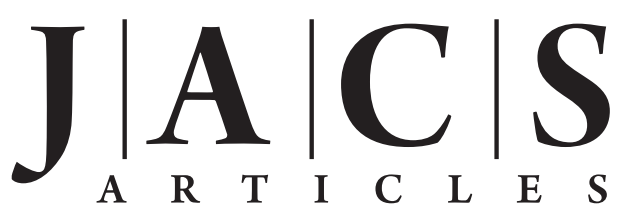

Published on Web 01/26/2009

\title{
Tetrapod-Shaped Colloidal Nanocrystals of II-VI Semiconductors Prepared by Seeded Growth
}

\author{
Angela Fiore, ${ }^{\dagger}$ Rosanna Mastria, ${ }^{\dagger}$ Maria Grazia Lupo, ${ }^{\dagger, \ddagger}$ Guglielmo Lanzani, ${ }^{\ddagger, \perp}$ \\ Cinzia Giannini, ${ }^{\S}$ Elvio Carlino," Giovanni Morello, ${ }^{\dagger}$ Milena De Giorgi, ${ }^{\dagger}$ Yanqin Li, ${ }^{\dagger}$ \\ Roberto Cingolani, ${ }^{\dagger}$ and Liberato Manna*,† \\ National Nanotechnology Laboratory (NNL) of CNR-INFM, Scuola Superiore ISUFI and IIT \\ Research Unit, Via per Arnesano 16, Km 5, 73100 Lecce, Italy, Politecnico di Milano, Piazza L. \\ Da Vinci 32, 20133 Milano, Italy, CNR-Istituto di Cristallografia (IC), via Amendola 122/O, \\ 70126 Bari, Italy, TASC-INFM-CNR National Laboratory, Area Science Park-Basovizza, \\ 34012 Trieste, Italy, and Fondazione Istituto Italiano di Tecnologia, Via Morego 30, \\ 16163 Genova, Italy
}

Received October 6, 2008; E-mail: liberato.manna@unile.it

\begin{abstract}
We report a general synthetic approach to tetrapod-shaped colloidal nanocrystals made of various combinations of $\mathrm{II}-\mathrm{VI}$ semiconductors. Uniform tetrapods were prepared using preformed seeds in the sphalerite structure, onto which arms were grown by coinjection of the seeds and chemical precursors into a hot mixture of surfactants. By this approach, a wide variety of core materials could be chosen (in practice, most of the II-VI semiconductors that could be prepared in the sphalerite phase, namely, CdSe, ZnTe, $\mathrm{CdTe}$ ); in contrast, the best materials for arm growth were CdS and CdTe. The samples were extensively characterized with the aid of several techniques.
\end{abstract}

\section{Introduction}

Solution-grown, tetrapod-shaped nanocrystals of various materials have attracted considerable interest in recent years because of their optical/electronic ${ }^{1-13}$ and mechanical properties $^{14}$ and chemical reactivities ${ }^{15,16}$ and hence for their potential

$†$ Scuola Superiore ISUFI and IIT Research Unit.

Politecnico di Milano.

$\$$ CNR-Istituto di Cristallografia.

"TASC-INFM-CNR National Laboratory.

${ }^{\perp}$ Fondazione Istituto Italiano di Tecnologia

(1) Jun, Y. W.; Lee, S. M.; Kang, N. J.; Cheon, J. J. Am. Chem. Soc. 2001, 123, 5150-5151.

(2) Bunge, S. D.; Krueger, K. M.; Boyle, T. J.; Rodriguez, M. A.; Headley, T. J.; Colvin, V. L. J. Mater. Chem. 2003, 13, 1705-1709.

(3) Manna, L.; Milliron, D. J.; Meisel, A.; Scher, E. C.; Alivisatos, A. P. Nat. Mater. 2003, 2 (6), 382-385.

(4) Chen, S. H.; Wang, Z. L.; Ballato, J.; Foulger, S. H.; Carroll, D. L. J. Am. Chem. Soc. 2003, 125, 16186-16187.

(5) Li, Y. C.; Zhong, H. Z.; Li, R.; Zhou, Y.; Yang, C. H.; Li, Y. F. Adv. Funct. Mater. 2006, 16, 1705-1716.

(6) Kumar, S.; Nann, T. Small 2006, 2, 316-329.

(7) Zhang, Y. L.; Zhong, X. H.; Zhu, J.; Song, X. Nanotechnology 2007, $18,195605$.

(8) Na, Y. J.; Kim, H. S.; Park, J. J. Phys. Chem. C 2008, 112, 11218 11226.

(9) Peng, P.; Milliron, D. J.; Hughes, S. M.; Johnson, J. C.; Alivisatos, A. P.; Saykally, R. J. Nano Lett. 2005, 5, 1809-1813.

(10) Pang, Q.; Zhao, L. J.; Cai, Y.; Nguyen, D. P.; Regnault, N.; Wang, N.; Yang, S. H.; Ge, W. K.; Ferreira, R.; Bastard, G.; Wang, J. N. Chem. Mater. 2005, 17, 5263-5267.

(11) Wang, L. W. J. Phys. Chem. B 2005, 109, 23330-23335.

(12) Malkmus, S.; Kudera, S.; Manna, L.; Parak, W. J.; Braun, M. J. Phys. Chem. B 2006, 110, 17334-17338.

(13) Al Salman, A.; Tortschanoff, A.; Mohamed, M. B.; Tonti, D.; van Mourik, F.; Chergui, M. Appl. Phys. Lett. 2007, 90, 093104.

(14) Fang, L.; Park, J. Y.; Cui, Y.; Alivisatos, P.; Shcrier, J.; Lee, B.; Wang, L. W.: Salmeron, M. J. Chem. Phys. 2007, 127, 184704

(15) Liu, H. T.; Alivisatos, A. P. Nano Lett. 2004, 4, 2397-2401. applications in fields such as photovoltaics, ${ }^{17-20}$ single-nanoparticle transistors, ${ }^{21}$ electromechanical devices, ${ }^{14}$ and recently also in scanning probe microscopy. ${ }^{22}$ In semiconductors such as those belonging to the II-VI group, the most credited and simplest explanation for the formation of these nanocrystals (both in solution-phase and gas-phase approaches) is that they nucleate in the cubic sphalerite phase, after which at some point the size evolution continues in the hexagonal phase. ${ }^{3,23-27}$ Because of the intrinsic similarities between the sphalerite and wurtzite structures, the fast growth of wurtzite domains that takes place along four out of the eight 111 crystallographic directions of a sphalerite nucleus does not generate strain at each sphalerite core-wurtzite arm interface. This is the case because along these directions there is a perfect match in lattice parameter between the two structures, and the only relevant structural difference between them is a change in the stacking sequence of atomic planes. In other materials, such as iron oxide, ${ }^{28}$ copper oxide, ${ }^{29}$

(16) Mokari, T.; Rothenberg, E.; Popov, I.; Costi, R.; Banin, U. Science 2004, 304, 1787-1790.

(17) Sun, B. Q.; Marx, E.; Greenham, N. C. Nano Lett. 2003, 3, 961-963.

(18) Zhou, Y.; Li, Y. C.; Zhong, H. Z.; Hou, J. H.; Ding, Y. Q.; Yang, C. H.; Li, Y. F. Nanotechnology 2006, 17, 4041-4047.

(19) Zhong, H. Z.; Zhou, Y.; Yang, Y.; Yang, C. H.; Li, Y. F. J. Phys. Chem. C 2007, 111, 6538-6543.

(20) Gur, I.; Fromer, N. A.; Chen, C. P.; Kanaras, A. G.; Alivisatos, A. P. Nano Lett. 2007, 7, 409-414.

(21) Cui, Y.; Banin, U.; Bjork, M. T.; Alivisatos, A. P. Nano Lett. 2005, $5,1519-1523$.

(22) Nobile, C.; Ashby, P. D.; Schuck, P. J.; Fiore, A.; Mastria, R.; Cingolani, R.; Manna, L.; Krahne, R. Small 2008, 4, 2123-2126.

(23) Manna, L.; Scher, E. C.; Alivisatos, A. P. J. Am. Chem. Soc. 2000, $122,12700-12706$

(24) Peng, Z. A.; Peng, X. G. J. Am. Chem. Soc. 2001, 123, 183-184.

(25) Yu, W. W.; Wang, Y. A.; Peng, X. G. Chem. Mater. 2003, 15, 43004308. 
and lead selenide, ${ }^{8}$ tetrapods are on the other hand single crystals (i.e., there is no difference between the central region and the arms with respect to either crystal structure or crystallographic orientation). In the latter cases, this peculiar shape can be explained as arising from the fastest growth rate of reactive corners present on the initially formed crystals, since they can protrude into regions of higher monomer concentration within the monomer diffusion layer that surrounds each nanocrystal. This may possibly cause the areas close to the corners (and sometimes edges) to grow much faster than other areas of the nanocrystal facets, such as their more central regions, leading to branched shapes. ${ }^{29}$ Additionally, faster consumption of monomers near the branches should intensify monomer diffusion toward these regions, thus further promoting their growth (this can be interpreted according to the so-called Mullins-Sekerka instability). ${ }^{30}$

Here we will confine our discussion to colloidal nanocrystals of II-VI semiconductors. Despite the appearance of several reports on tetrapod-shaped nanocrystals of these materials in recent years, ${ }^{2,3,5,25,31-33}$ the fabrication of such nanoparticle shapes in high yields in the liquid phase is notoriously difficult because of the inherent mechanism of their formation. Many syntheses indeed yield mixtures of rods, dipods, tripods, tetrapods, and even hyper-branched nanoparticles. This is probably due to the fact that one cannot strictly identify reaction conditions that promote nucleation entirely in the cubic sphalerite phase and growth entirely in the hexagonal wurtzite phase. For example, if nucleation of both wurtzite and sphalerite nuclei takes place, the final sample will be seriously contaminated with rods. In addition to this, concerted growth of arms out of a nucleus often does not take place, and therefore, even in samples rich in tetrapods, there is a considerable distribution of arm lengths. This should become an issue when, for instance, tetrapods are used in photovoltaic devices, which ideally would require a uniform film of tetrapods, each having three arms touching a substrate (acting as one of the electrodes) and the fourth arm pointing upward and spanning the whole thickness of the active layer in the cell.

One way of getting around these problems is via a "seededgrowth" approach. Seeded growth of shape-controlled colloidal nanocrystals is a well-established procedure, especially for metals, ${ }^{34-37}$ and has been exploited even to grow star-shaped Au nanocrystals starting from multiple-twinned Au nanoparticles as seeds. ${ }^{38}$ The major advantage of the method is that it

(26) Gong, J. F.; Yang, S. G.; Huang, H. B.; Duan, J.; Liu, H. W.; Zhao, X. N.; Zhang, R.; Du, Y. W. Small 2006, 2, 732-735.

(27) Ding, Y.; Wang, Z. L.; Sun, T. J.; Qiu, J. S. Appl. Phys. Lett. 2007, 90, 153510.

(28) Cozzoli, P. D.; Snoeck, E.; Garcia, M. A.; Giannini, C.; Guagliardi, A.; Cervellino, A.; Gozzo, F.; Hernando, A.; Achterhold, K.; Ciobanu, N.; Parak, F. G.; Cingolani, R.; Manna, L. Nano Lett. 2006, 6, 19661972.

(29) Xu, J. S.; Xue, D. F. Acta Mater. 2007, 55, 2397-2406.

(30) Mullins, W. W.; Sekerka, R. F. J. Appl. Phys. 1964, 35, 444.

(31) Zhang, J. Y.; Yu, W. W. Appl. Phys. Lett. 2006, 89, 123108.

(32) Carbone, L.; Kudera, S.; Carlino, E.; Parak, W. J.; Giannini, C.; Cingolani, R.; Manna, L. J. Am. Chem. Soc. 2006, 128, 748-755.

(33) Asokan, S.; Krueger, K. M.; Colvin, V. L.; Wong, M. S. Small 2007, 3, 1164-1169.

(34) Jana, N. R.; Gearheart, L.; Murphy, C. J. Adv. Mater. 2001, 13, 13891393.

(35) Jana, N. R.; Gearheart, L.; Murphy, C. J. J. Phys. Chem. B 2001, $105,4065-4067$.

(36) Nikoobakht, B.; El-Sayed, M. A. Chem. Mater. 2003, 15, 1957-1962.

(37) Habas, S. E.; Lee, H.; Radmilovic, V.; Somorjai, G. A.; Yang, P. Nat. Mater. 2007, 6, 692-697.

(38) Nehl, C. L.; Liao, H.; Hafner, J. H. Nano Lett. 2006, 6, 683-686.
Scheme 1. Sketch Highlighting the Seeded-Growth Approach to Tetrapod-Shaped Nanocrystals Based on a Combination of Different Materials for the Core and Arms

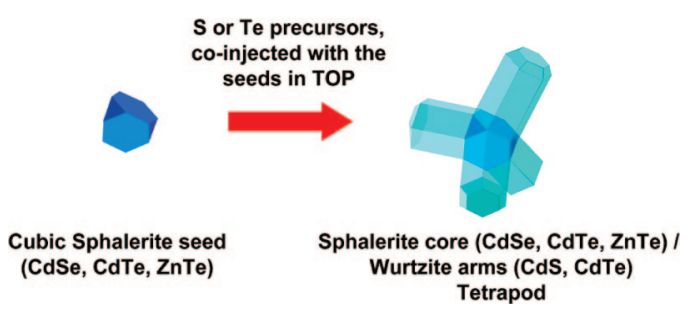

overcomes the nucleation stage with all its associated problems of overlap of nucleation with growth that inevitably lead to broad distributions of sizes and shapes. To date, this approach has been reported by a few groups (including ours) for the preparation of $\mathrm{ZnTe} / \mathrm{CdSe},{ }^{39} \mathrm{ZnTe} / \mathrm{CdS},{ }^{39,40} \mathrm{ZnSe} / \mathrm{CdS},{ }^{40}$ and $\mathrm{CdSe} / \mathrm{CdS}^{41}$ tetrapods (where the first compound listed is the material of the core and the second that of the arms). In such cases, preformed nuclei in the sphalerite phase are coinjected with the precursors needed for growth of the tetrapod arms into a hot mixture of surfactants that promotes wurtzite growth. ${ }^{39-41}$ A more controlled and "concerted" growth of wurtzite arms on top of such seeds is usually observed (especially when large seeds are employed), and this favors the formation of arms with more uniform lengths in each tetrapod. Furthermore, the material of the seed and that of the arms can be clearly different, which yields tetrapod structures with more tunable properties than those traditionally formed from a single material. More recent methods for improving the yield of tetrapods include seeded growth starting from noble metal nanoparticles ${ }^{42}$ and, in the case of $\mathrm{CdS}$ nanocrystals, the coinjection of $\mathrm{Se}$ or $\mathrm{Te}$ precursors to enhance the probability of formation of sphalerite nuclei at the early stages of tetrapod formation. ${ }^{43}$

In addition to tetrapods, many branched heterostructured nanocrystals have been prepared and studied to date by several groups, although the various nanocrystals were not prepared with a properly identifiable seeded-growth approach (i.e., by coinjection of preformed seeds),,${ }^{9,19,44}$ and additionally, those reports mainly aimed at exploring the optical and electronic properties of such nanocrystals rather than at achieving monodispersity in shape.

Here we report a more general approach for synthesizing tetrapod-shaped colloidal nanocrystals made of various combinations of II-VI semiconductors, using preformed seeds in the sphalerite structure on which mainly hexagonal wurtzite arms are formed by coinjection of the seeds and chemical precursors into a hot mixture of surfactants (see Scheme 1). For the core region of the tetrapod and hence the seed, we could choose among CdSe, ZnTe, and CdTe, as nanocrystals of these materials could be prepared in the sphalerite phase and also because they gave good yields in terms of tetrapods when used as seeds; on the other hand, the best materials for arm growth were $\mathrm{CdS}$ and CdTe. The nanocrystals were characterized using several techniques, including various microscopy analyses, X-ray dif-

(39) Xie, R. G.; Kolb, U.; Basché, T. Small 2006, 2, 1454-1457.

(40) Carbone, L.; et al. Nano Lett. 2007, 7, 2942-2950.

(41) Talapin, D. V.; Nelson, J. H.; Shevchenko, E. V.; Aloni, S.; Sadtler, B.; Alivisatos, A. P. Nano Lett. 2007, 7, 2951-2959.

(42) Yong, K. T.; Sahoo, Y.; Swihart, M. T.; Prasad, P. N. Adv. Mater. 2006, 18, 1978-1982.

(43) Hsu, Y. S.; Lu, S. Y. Small 2008, 4, 951-955.

(44) Milliron, D. J.; Hughes, S. M.; Cui, Y.; Manna, L.; Li, J. B.; Wang, L. W.; Alivisatos, A. P. Nature 2004, 430, 190-195. 
Table 1. Summary of All of the Parameters Involved in the Syntheses of the Tetrapod-Shaped Nanocrystals Shown in Figure 1

\begin{tabular}{llllll}
\hline & \multicolumn{1}{c}{ ZnTe/CdS } & ZnTe/CdTe & ZnTe/CdSe & CdSe/CdTe \\
\hline amount of seeds $(\mu \mathrm{mol})$ & $9.6 \times 10^{-3}$ & $9.6 \times 10^{-3}$ & $19 \times 10^{-3}$ & $26 \times 10^{-3}$ & $51 \times 10^{-3}$ \\
precursor solution $^{a}$ & $\mathrm{~S} / \mathrm{TOP}, 1.5 \mathrm{~g}$ & $\mathrm{Te} / \mathrm{TOP}, 0.6 \mathrm{~g}$ & $\mathrm{Se} / \mathrm{TOP}, 0.5 \mathrm{~g}$ & $\mathrm{Te} / \mathrm{TOP}, 0.6 \mathrm{~g}$ & $\mathrm{Te} / \mathrm{TOP}, 0.6 \mathrm{~g}$ \\
$T_{\text {injection }}\left({ }^{\circ} \mathrm{C}\right)$ & 350 & 320 & 320 & 340 & 320 \\
growth time $(\mathrm{min})$ & 7 & 3 & 7 & 3 & 3 \\
\hline
\end{tabular}

${ }^{a}$ Solution concentrations: S/TOP, $67 \mathrm{mg} / \mathrm{mL}$; Te/TOP, $10 \mathrm{wt} \%$; Se/TOP, $12 \mathrm{mg} / \mathrm{mL}$.

fraction (XRD), and pump-and-probe and photoluminescence spectroscopy, which aimed at assessing both their structural and optical features.

\section{Experimental Section}

2.1. Chemicals and Stock Solutions. Trioctylphosphine oxide (TOPO, 99\%), trioctylphosphine (TOP, 97\%), cadmium stearate (90\%), sulfur (99\%), tellurium (99.999\%), selenium (99.99\%), and diethylzinc $\left(\mathrm{Et}_{2} \mathrm{Zn}, 97 \%\right)$ were purchased from Strem Chemical. $\mathrm{Et}_{2} \mathrm{Zn}$ was distilled under vacuum prior to use. Octadecylphosphonic acid (ODPA, 99\%) and hexylphosphonic acid (HPA, 99\%) were purchased from Polycarbon Industries. Cadmium oxide $(\mathrm{CdO}$, 99.5\%), octadecylamine (ODA, 97\%), 1-octadecene (ODE, 90\%), oleic acid $(90 \%)$, and oleylamine $(70 \%)$ were purchased from Sigma-Aldrich. TOPO (90\%) was purchased from Alfa Aesar. All of the solvents were anhydrous and were purchased from SigmaAldrich. A 10 wt \% stock solution of Te in TOP was prepared by weighing $1 \mathrm{~g}$ of pure Te powder in a three-neck flask, adding to it $9 \mathrm{~g}$ of TOP, and then heating the mixture at $320^{\circ} \mathrm{C}$ with vigorous stirring under a nitrogen flow. Stock solutions of Se in TOP (12 $\mathrm{mg} / \mathrm{mL})$ and $\mathrm{S}$ in TOP $(67 \mathrm{mg} / \mathrm{mL})$ were prepared in a glovebox at room temperature.

2.2. Synthesis and Purification of Nanocrystal Seeds and Estimation of Their Concentration. All of the syntheses of nanocrystals used for subsequent growth of tetrapods (henceforth called "seeds") were carried out under an inert atmosphere using standard air-free techniques. After each of the syntheses, which are described in detail in the following sections, $\mathrm{ZnTe}$ and $\mathrm{CdSe}$ seeds were precipitated with isopropyl alcohol, while CdTe seeds were precipitated with methanol. They were then washed by repeated redissolution in toluene and precipitation with methanol and finally dissolved in TOP. For each sample of seeds, the concentration of nanocrystals in TOP was adjusted to fit the optimized amount of seeds to be injected for the synthesis of tetrapods. The concentration of seeds was determined in the following way: First, the average nanocrystal diameter was assessed via statistical analysis on transmission electron microscopy (TEM) images of several hundreds of nanocrystals (see below for details on TEM). Second, the average number of atoms of one atomic species per nanocrystal (e.g., Zn for ZnTe) was determined by building a structural model of the nanocrystal with the diameter as determined by TEM. Finally, the total concentration of that atomic species in the solution of nanocrystals was then estimated via elemental analysis (see details below for setups and procedures). Knowledge of the average number of a given atomic species per nanocrystal and of its total concentration in solution allowed for the determination of the concentration of nanocrystals.

A. Synthesis of ZnTe Seeds. ZnTe nanocrystals with the sphalerite structure were prepared according to a previously reported procedure with minor changes. ${ }^{39,45}$ In a three-neck flask, ODA (1 g) and $\operatorname{ODE}(3 \mathrm{~g})$ were mixed, heated to $\sim 150^{\circ} \mathrm{C}$, and pumped to vacuum for $\sim 1 \mathrm{~h}$. The solution was then heated to $280^{\circ} \mathrm{C}$ under a nitrogen flow. A solution of Te and $\mathrm{Et}_{2} \mathrm{Zn}$ in TOP $(0.1 \mathrm{~g}$ of $10 \mathrm{wt}$ $\% \mathrm{Te} / \mathrm{TOP}$ stock solution $+0.096 \mathrm{~g}$ of $\mathrm{Et}_{2} \mathrm{Zn}+0.9 \mathrm{~g}$ of TOP, $\mathrm{Te} / \mathrm{Zn}$ molar ratio $=1: 1$ ) was injected quickly into the reaction flask. After the injection, the reaction temperature was set to 270 ${ }^{\circ} \mathrm{C}$, and the nanocrystals were allowed to grow for $3 \mathrm{~min}$.

(45) Xie, R. G.; Zhong, X. H.; Basché, T. Adv. Mater. 2005, 17, 27412745.
B. Synthesis of CdSe Seeds. CdSe seeds with the sphalerite structure were synthesized by a modification of a published procedure. ${ }^{41} \mathrm{In}$ a $100 \mathrm{~mL}$ three-neck flask, $0.48 \mathrm{~g}(0.6 \mathrm{mmol})$ of cadmium stearate was dissolved in $37 \mathrm{~mL}$ of ODE and pumped to vacuum at $90{ }^{\circ} \mathrm{C}$ for $40 \mathrm{~min}$. The reaction mixture was cooled to room temperature, and $0.024 \mathrm{~g}(0.3 \mathrm{mmol})$ of Se powder was added to the reaction mixture, which was then degassed under vacuum for $10 \mathrm{~min}$ at $50{ }^{\circ} \mathrm{C}$. The solution was heated to $240{ }^{\circ} \mathrm{C} ; 3 \mathrm{~min}$ after the mixture approached $240{ }^{\circ} \mathrm{C}$, a degassed solution of 0.1 $\mathrm{mL}$ of oleic acid and $1 \mathrm{~mL}$ of oleylamine in $4 \mathrm{~mL}$ of ODE was added dropwise to the reaction mixture to stabilize the nanoparticle growth. After $1 \mathrm{~h}$ of reaction, the heating was stopped.

C. Synthesis of CdTe Seeds. To synthesize CdTe nanocrystals with the sphalerite structure, a mixture of 90\% TOPO (3.0 g), ODPA $(0.315 \mathrm{~g})$, and $\mathrm{CdO}(60 \mathrm{mg})$ was loaded into a $50 \mathrm{~mL}$ threeneck flask and degassed for $90 \mathrm{~min}$ at $180{ }^{\circ} \mathrm{C}$. Next, under nitrogen flow, the mixture was heated to $380{ }^{\circ} \mathrm{C}$ and held at that temperature until the solution turned clear. At this point, $1.5 \mathrm{~g}$ of TOP was added, and the temperature was allowed to recover to $380^{\circ} \mathrm{C}$, after which $0.780 \mathrm{~g}$ of the $10 \mathrm{wt} \% \mathrm{Te} / \mathrm{TOP}$ stock solution was quickly injected. Immediately after the injection, the heating mantle was removed.

2.3. Synthesis of Tetrapod-Shaped Heterostructures with ZnTe, CdSe, or CdTe Cores and CdX (X = S, Te, Se) Arms. For a typical synthesis of heterostructured tetrapods, 99\% TOPO (3.0 g), ODPA (0.290 g), HPA (0.080 g), and CdO (0.060 g) were mixed in a $50 \mathrm{~mL}$ flask. After the flask was pumped to vacuum for $\sim 1 \mathrm{~h}$ at $150{ }^{\circ} \mathrm{C}$, the resulting solution was heated to $320-350{ }^{\circ} \mathrm{C}$ (depending on the specific synthesis) under nitrogen to yield a colorless, clear solution. At this point, $1.5 \mathrm{~g}$ of TOP was injected in the flask, and the temperature was allowed to recover to the value required for the synthesis. The solution of precursors plus nanocrystal seeds was prepared by mixing a specified amount of sulfur (or tellurium or selenium) stock solution with the solution of nanocrystal seeds (ZnTe, CdSe, or CdTe). This solution was quickly injected into the flask, and then the system was allowed to react for a given time (see Table 1). The final sample was washed by repeated precipitation via addition of methanol and redissolution in toluene. The as-synthesized heterostructures often contained small amounts of byproducts such as nanorods, dipods, and tripods. Most of them could be separated from the tetrapods by size/shapeselective precipitation. We added some drops of methanol and centrifuged the resultant solution. The precipitate that formed contained almost exclusively tetrapods. It was separated from the supernatant and redissolved in toluene. $\mathrm{ZnTe} / \mathrm{CdX}(\mathrm{X}=\mathrm{S}, \mathrm{Te}, \mathrm{Se})$ as well as $\mathrm{CdSe} / \mathrm{CdTe}$ and $\mathrm{CdTe} / \mathrm{CdTe}$ nanocrystals were synthesized according to the above procedure, with some differences among them depending on the heterostructure (see Table 1 for amounts and conditions). Here and in all of the following discussions, the notation " $\mathrm{A} / \mathrm{B}$ " indicates that material " $\mathrm{A}$ " is that of the core (i.e., the starting seed) and material " $\mathrm{B}$ " is that of the arms.

Several control syntheses were carried out in order to fully identify the roles of the seeds and additional parameters (such as the composition of the mixture of surfactants and the growth temperature) in the formation of the heterostructures. In regard to the seeds, we repeated the same syntheses as reported above for growing $\mathrm{CdX}$ arms $(\mathrm{X}=\mathrm{S}, \mathrm{Te}, \mathrm{Se})$ on the seeds but with no seeds coinjected. Additionally, we repeated the various syntheses using as seeds CdSe nanocrystals with the wurtzite structure $(\mathrm{CdSe}$ is the only material for which we could synthesize at will seeds with 
either the wurtzite or sphalerite structure). In regard to the mixture of surfactants, we performed representative control syntheses in which we attempted to grow $\mathrm{ZnTe} / \mathrm{CdTe}$ and $\mathrm{CdSe} / \mathrm{CdTe}$ heterostructures using either HPA or ODPA only (instead of a mixture of them, as discussed above) in addition to TOPO, with the ratio of phosphonic acids to $\mathrm{Cd}$ maintained the same as in the previous syntheses. Finally, we performed representative control syntheses of tetrapods at growth temperatures lower than those reported in Table 1. For instance, $\mathrm{ZnTe} / \mathrm{CdTe}$ tetrapods were also grown at $280{ }^{\circ} \mathrm{C}$ (instead of $320{ }^{\circ} \mathrm{C}$ ) and $\mathrm{ZnTe} / \mathrm{CdS}$ tetrapods at $300{ }^{\circ} \mathrm{C}$ (instead of $350{ }^{\circ} \mathrm{C}$ ).

2.4. Optical Characterization. The optical absorption measurements on the various samples (either on aliquots of the growth solutions taken during the various syntheses and further diluted in toluene or on the final purified samples) were performed using a Varian Cary 300 UV-vis spectrophotometer. Room-temperature photoluminescence (PL) spectra of the various seeds dissolved in toluene were recorded on a Varian Cary Eclipse fluorescence spectrophotometer. The PL spectra of the various tetrapods, on the other hand, were recorded at low temperatures $(13 \mathrm{~K})$ in both the visible and infrared regions. The nanocrystals were deposited by drop-casting from toluene solutions on $\mathrm{Si} / \mathrm{SiO}_{2}$ substrates and placed in vacuum in a closed-cycle He cryostat. The samples were excited using an argon ion laser $(\lambda=514 \mathrm{~nm})$. The PL signal was dispersed by a monochromator and detected by a Si CCD detector.

Tetrapods with cores made of either ZnTe, CdSe, or CdTe and arms made of CdTe were additionally investigated by means of pump-and-probe spectroscopy in the visible spectral range in order to understand electron-hole dynamics and identify the role of the different interfaces (type II for CdSe/CdTe and "all-CdTe" tetrapods and type I for $\mathrm{ZnTe} / \mathrm{CdTe}$ tetrapods). The experimental setup was the same as that described by Polli and co-workers in a published work. ${ }^{46}$ All of the measurements were performed on solutions of tetrapods dispersed in toluene at room temperature, and the geometry employed the magic angle between the pump and probe polarizations in order to exclude transient anisotropy effects on the dynamics. ${ }^{12,47}$ The pump energy was $3.2 \mathrm{eV}(390 \mathrm{~nm})$, and the pump fluence was $700 \mu \mathrm{J} / \mathrm{cm}^{2}$.

2.5. Compositional Analysis of Nanocrystal Ensembles. An inductively coupled plasma atomic emission spectrometer (Varian Vista $\mathrm{AX}$ ) was used to investigate the elemental composition of the nanocrystals. The samples were digested in 3:1 (v/v) $\mathrm{HCl} / \mathrm{HNO}_{3}$ using a CEM MARS 5 microwave digester.

2.6. Structural Characterization by TEM/STEM/EDS. The samples were prepared by dispersing dilute solutions of nanocrystals onto carbon-coated copper grids. Low-magnification TEM images were recorded on a Jeol JEM 1011 microscope operated at 100 kV. High-magnification TEM, scanning TEM (STEM), and energydispersive X-ray spectroscopy (EDS) experiments were performed at room temperature on a Jeol JEM 2010F UHR microscope equipped with an Oxford EDS $\mathrm{Si}(\mathrm{Li})$ spectrometer and a fieldemission Shottky cathode. All of the experiments were performed at an accelerating voltage of $200 \mathrm{kV}$, corresponding to an electron wavelength of $0.025 \mathrm{~nm}$. The equipment had a low-sphericalaberration objective lens (spherical aberration coefficient $C_{\mathrm{s}}=0.47$ $\pm 0.01 \mathrm{~mm}$ ) and a relevant resolution at optimum defocus in phasecontrast high-resolution TEM (HRTEM) of $0.19 \mathrm{~nm} .{ }^{48}$ STEM highangle annular dark field (HAADF) images were acquired using an annular detector with a collection angle of $84 \mathrm{mrad} \leq 2 \theta \leq 224$ mrad. With this configuration, the attainable resolution in HAADF imaging at optimum defocus was $0.12 \mathrm{~nm} .{ }^{49}$ HAADF imaging is

(46) Polli, D.; Lüer, L.; Cerullo, G. Rev. Sci. Instrum. 2007, 78, 103108. (47) Gadermaier, C.; Lanzani, G. J. Phys.: Condens. Matter 2002, 14, $9785-$ 9802.

(48) Spence, J. C. H. In Experimental High-Resolution Electron Microscopy, 2nd ed.; Oxford University Press: Oxford, U.K., 1988; p 87.

(49) Carlino, E.; Grillo, V. Proceedings of the Seventh Multinational Congress on Microscopy - MCM 2005, Portoroz, Slovenia, June 2630, 2005; p 159. strongly sensitive to the atomic number of the specimen (this technique is also referred to as "Z-contrast imaging"). The sensitivity of HAADF imaging can be exploited to achieve atomic-resolution information on the chemical composition of the sample..$^{50-52}$ The EDS experiments were performed in the STEM configuration by scanning an $\sim 0.2 \mathrm{~nm}$ electron probe along the tetrapod and acquiring the relevant characteristic X-ray spectrum. During data acquisition, a specimen drift corrector actively checked for possible drift every $60 \mathrm{~s}$. To achieve reasonable statistics in the spectra, it was necessary to work with acquisition times on the order of thousands of seconds. Hence, special care was taken to minimize the drift and hydrocarbon contamination of the specimen and specimen holder by following a well-established procedure published elsewere. ${ }^{53}$

2.7. XRD Experiments and Simulations. The powder XRD measurements were performed with a RIGAKU-INEL powder diffractometer with a $12 \mathrm{~kW}$ rotating anode (RINT200 series) and a copper anode, a germanium (111) monochromator, and a curved position-sensitive detector (CPS-120 INEL). In most cases, concentrated solutions of the purified nanocrystals were spread on top of a miscut silicon substrate. The samples were allowed to dry and then measured in the reflection geometry. However, in the case of ZnTe nanocrystals, which were found to degrade quickly upon exposure to air, measurements were carried out on powders sealed in quartz capillaries. The diffraction patterns of the seeds were fitted with the program FullProf, ${ }^{54}$ which is based on a Rietveld refinement approach. Full simulations of the powder diffraction patterns from tetrapods were performed instead with a Debye function-based method. The following steps were needed in order to simulate the pattern: (1) generation of a structural model of the nanocrystal; (2) computation of the corresponding database of interatomic distances; (3) sampling of the interatomic distances; and finally (4) calculation of the diffraction pattern. The sampling of the interatomic distances was necessary because their number increased exponentially with the cluster size and quickly reached billions of distances. In the Gaussian sampling that we adopted, each distance was convoluted in direct space with a Gaussian function. All of these Gaussians were then summed to obtain a continuous atomic pair distance distribution function, which was then sampled on a fixed grid with a suitable step $\Delta(\Delta<\rho$, where $\rho$ is the Gaussian width). ${ }^{55}$ Once the models and the corresponding sampled pair distribution function were available, the diffraction pattern (apart from several simple additive and multiplicative corrections) was calculated according to the Debye formula:

$$
I(q)=\sum_{k=1}^{M} \mu_{k} \frac{\sin \left(2 \pi q d_{k}\right)}{2 \pi q d_{k}}
$$

where $q$ is the scattering vector and $d_{k}$ and $\mu_{k}$ are the sampled interatomic distances and relative multiplicities, respectively.

\section{Results and Discussion}

3.1. Synthesis and Characterization of the Tetrapods by Electron Microscopy. Figure 1 reports low-magnification TEM images of various samples of tetrapod-shaped nanocrystals prepared by this approach. The degree of homogeneity seen in the samples and the absence of the originally coinjected seeds suggest that the growth of the tetrapods indeed proceeded via

(50) Pennycook, S. J. Adv. Imaging Electron Phys. 2002, 123, 140.

(51) Yamazaki, T.; Watanabe, K.; Kikuchi, K.; Kawasaki, M.; Hashimoto, I.; Shiojiri, M. Phys. Rev. B 2000, 61, 13833.

(52) Carlino, E.; Grillo, V. Phys. Rev. B 2005, 71, 235303.

(53) Carlino, E.; Martelli, F.; Rubini, S.; Franciosi, A. Philos. Mag. Lett. 2006, 86, 261.

(54) FullProf Suite Web page. http://www.ill.eu/sites/fullprof/index.html (accessed Jan 12, 2009).

(55) Cervellino, A.; Giannini, C.; Guagliardi, A. J. Comput. Chem. 2006, 27, 995-1008. 


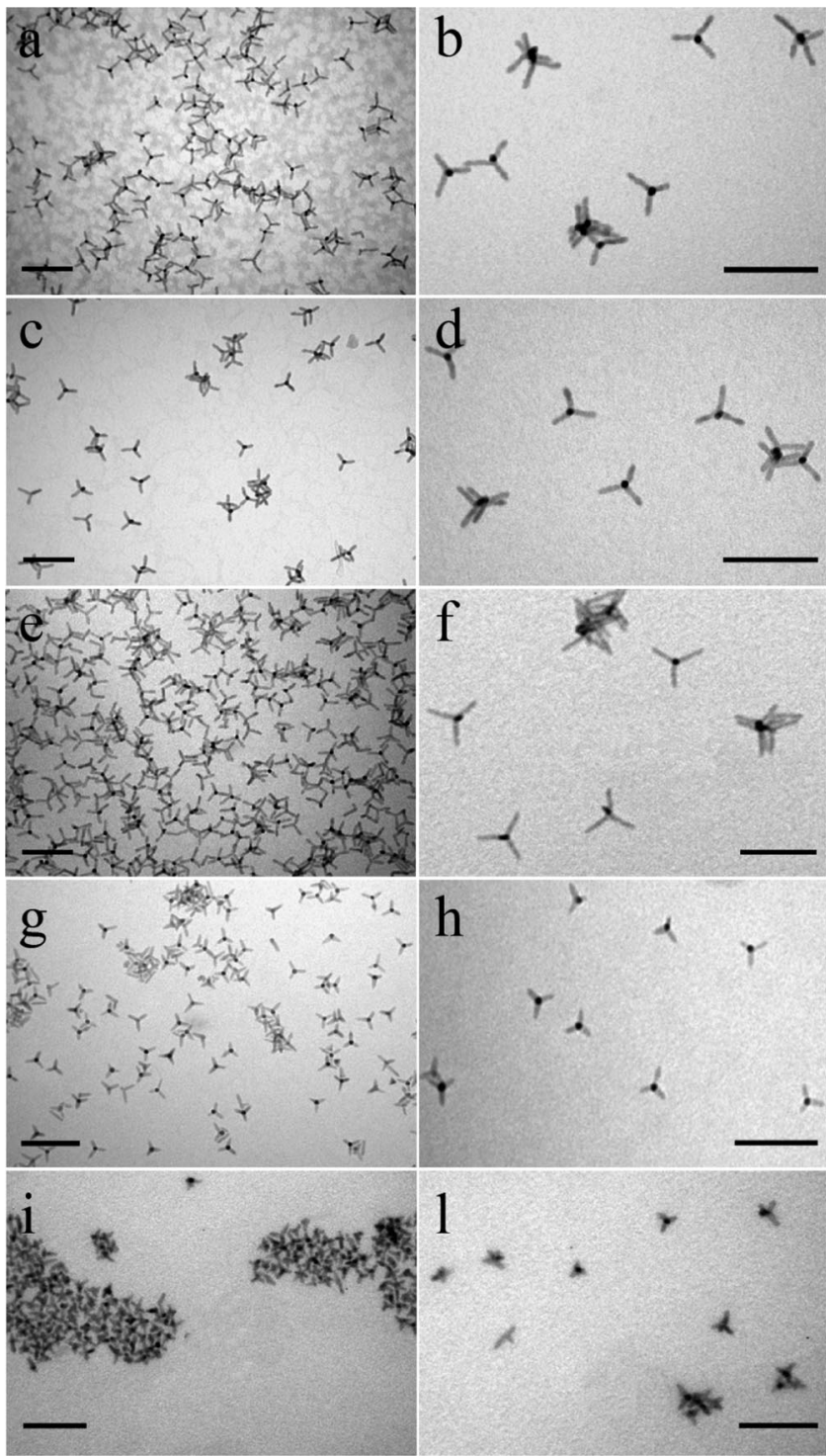

Figure 1. TEM images of tetrapod-shaped nanocrystals (denoted as core/ arm) prepared by the seeded-growth approach. From top to bottom, the panels refer to the following tetrapods: (a, b) CdTe/CdTe; (c, d) CdSe/ CdTe; (e, f) ZnTe/CdTe; (g, h) ZnTe/CdS; and (i, l) ZnTe/CdSe. All of the scale bars are $100 \mathrm{~nm}$ long. Each horizontal couple of panels shows two different TEM images of the same sample, but the right panel shows an area at a higher magnification than the left panel.

concerted growth of four arms on top of the seeds with high yields (i.e., via heterogeneous nucleation of the arm material rather than via homogeneous nucleation), regardless on the chemical nature of the seed (i.e., whether it was ZnTe, CdSe, or CdTe), and that basically all of the seeds were used up. The most suitable materials for arm growth were $\mathrm{CdS}$ and CdTe, for which the arm lengths could reach up to $50 \mathrm{~nm}$, whereas the growth of CdSe on various types of seeds led to tetrapod morphologies with arms that were no more than a few nanometers long and in most cases had sharpened tips (see, for instance, the images in Figure 1i,1 for $\mathrm{ZnTe} / \mathrm{CdSe}$ tetrapods, which are similar to those reported by the group of Basché ${ }^{39}$ ). Different reaction conditions (i.e., working at large ratios of $\mathrm{Cd} / \mathrm{Se}$ precursors, injecting a smaller number of seeds, carrying out the same synthesis at a different temperature, or modifying the relative ratios of surfactants) did not seem to lead to substantial improvement in the final product, as tetrapods with sharpened arms were formed again. Injecting more precursors for arm growth, for instance, led only to fatter nanocrystals, with shapes that more closely resembled stars than tetrapods. One explanation for this behavior could be that in the present surfactants, the growth rate of wurtzite $\mathrm{CdSe}$, as opposed to $\mathrm{CdS}$ and CdTe, along the 001 direction is so fast that the 001 facets are quickly replaced by slower-growing $11 l$ or $10 l$ facets $(l=1,2, \ldots)$, which effectively shut down the growth along the 001 direction. Indeed even when no seeds were injected, $\mathrm{CdSe}$ rods with irregular shapes and sharpened tips were formed (see below and also the Supporting Information). We therefore believe that growing longer and more uniform CdSe arms requires finding more suitable mixtures of surfactants that those used by us in the present work. However, the underlying principle of seeded growth is still valid for fabricating tetrapods with CdSe arms.

When the same syntheses as reported above for growing $\mathrm{CdX}$ $(\mathrm{X}=\mathrm{S}, \mathrm{Te}, \mathrm{Se})$ arms were repeated but with no seeds coinjected, the final samples were characterized by a wide variety of morphologies, including nanorods, bipods, and tripods but almost no tetrapods; additionally, broad distributions of arm lengths were observed (see Figure S1 in the Supporting Information for low-magnification TEM images of nanocrystals prepared without coinjection of seeds). Furthermore, when wurtzite CdSe seeds were used instead of sphalerite CdSe seeds, growth of $\mathrm{CdS}$ led to uniform rod-shaped nanocrystals (as reported previously by our group ${ }^{40}$ ) while growth of CdTe led to samples that consisted mainly in rods and dipods. The corresponding results of this latter synthesis are shown in Figure $\mathrm{S} 2$ in the Supporting Information. All of these control experiments clearly support the conclusion that coinjection of sphalerite seeds is needed in order to achieve homogeneous tetrapod growth.

The importance of working with an appropriate mixture of two different alkyl phosphonic acids (namely, HPA and ODPA, with an HPA/ODPA molar ratio of 0.5) in order to guarantee a high yield in tetrapods was stressed by the outcome of control syntheses of $\mathrm{ZnTe} / \mathrm{CdTe}$ and $\mathrm{CdSe} / \mathrm{CdTe}$ heterostructures in which only HPA or only ODPA was present instead of a mixture of the two acids. These syntheses yielded either roughly spherical nanocrystals (when ODPA was used) or highly defective and probably multicrystalline nanostructures (when HPA was used) (see Figure S3 in the Supporting Information). Additional control syntheses carried out at lower temperatures than those reported in Table 1 in general led to lower yields of tetrapods, and the tetrapods so obtained had shorter and more irregular arms (see Figure S4 the Supporting Information for results on $\mathrm{ZnTe} / \mathrm{CdTe}$ and $\mathrm{ZnTe} / \mathrm{CdS}$ heterostructures).

Extensive structural analysis via high magnification electron microscopy was carried out on these tetrapod samples. We report here only the relevant results for samples consisting of a ZnTe core and CdTe arms. In the TEM specimen, the large majority of the nanostructures were deposited on the carbon film with one of the arms oriented close to the electron-beam direction, and hence, imaging of the tetrapod did not help to characterize the core of the structure from the point of view of its chemical composition. Figure 2a shows an HRTEM image of a ZnTe/ CdTe tetrapod with the outgoing arm nearly parallel to the electron beam. The interpretation of the atomic-resolution HRTEM image contrast is particularly difficult in the case of the outgoing arm, as it is due to the combination of the projected potential of the CdTe arm along the [0001] zone axis of the wurtzite structure and the projected potential of the core of the 


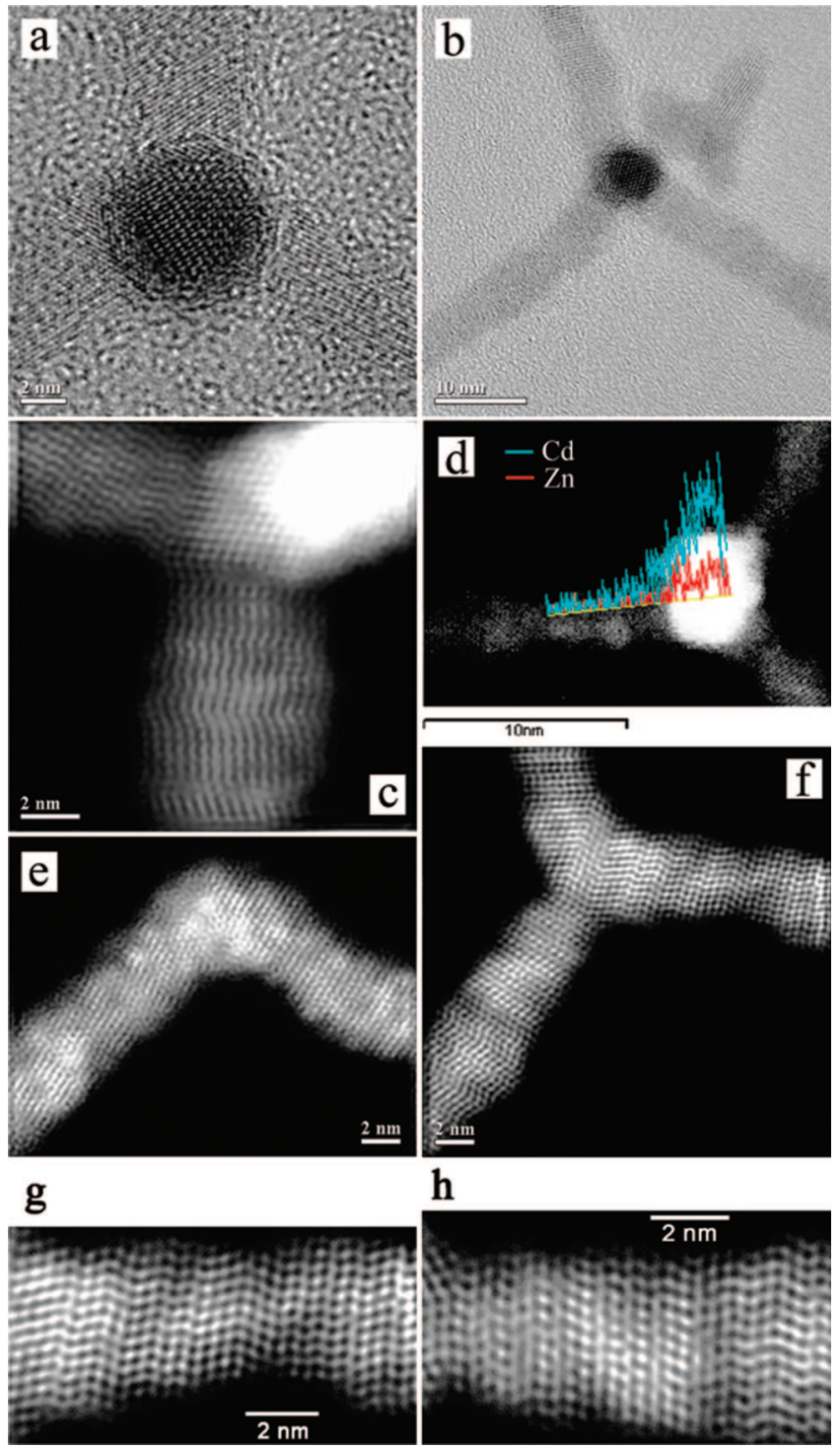

Figure 2. (a) HRTEM image of a $\mathrm{ZnTe} / \mathrm{CdTe}$ tetrapod with the outgoing arm oriented nearly parallel to the electron beam. (b) HRTEM image of a tetrapod and of a small tripod. It is remarkable to note the changes in the lattice-fringe contrast along the arms of the tetrapod, which indicates the presence of regions with either different orientations, structures, or compositions. (c) HAADF image of a tetrapod tilted to show some of the interfaces between the core region and the arms. (d) HAADF image and spatially resolved EDS spectra showing the presence of $\mathrm{Zn}$ in the core region of the tetrapod. It is worth noting that some counts of $\mathrm{Zn}$ above the background level come from regions of lower image intensity along the arm. (e, f) HAADF images of (e) a dipod and (f) a tripod. (g, h) Magnified views of the $(\mathrm{g})$ right and $(\mathrm{h})$ bottom arms of the tripod in (f).

tetrapod, all convoluted with the contrast-transfer function of the objective lens. ${ }^{48}$ Hence, it is not possible to obtain direct information on the core of the tetrapod even if the image is well-resolved and rich in detail. Figure 2b shows an HRTEM image of a tetrapod with the outgoing CdTe arm oriented close to the direction of the electron beam, together with a small tripod. This tetrapod has some features common to most of the observed structures characterized by CdTe arms, namely, the lattice-fringe image contrast often varies along the arms. This indicates the presence of multidomain regions, characterized either by different structures or chemical compositions or simply by different crystallographic orientations. In the case of tetrapods grown from ZnTe seeds, this could even be caused by the formation of alloys containing zinc, as also pointed by the
HAADF and EDS experiments (see below), as a result of the possible diffusion of this element into the CdTe arms (interdiffusion effects have also been observed recently in linear $\mathrm{CdTe} / \mathrm{CdSe} / \mathrm{CdTe}$ heterojunction nanorods ${ }^{56}$ ). In contrast, HRTEM images of tetrapods based on CdS arms (not shown here) reveal that the latter have much closer to single $\mathrm{CdS}$ wurtzite domains. Such differences between CdS and CdTe tetrapod arms in the various samples were confirmed by the XRD analysis (see below).

The contrast of the HAADF image is strongly related to the average atomic number of the specimen and to its thickness. ${ }^{50}$ Figure $2 \mathrm{c}$ is a HAADF image of a slightly tilted tetrapod that shows some details of the interface regions between the core and the arms of the tetrapod. The brighter area in the HAADF image is mostly due to the relatively high thickness of the outgoing arm, maximized in this geometrical configuration, which saturates the image contrast in this region. The thickness effect hides every possible contrast variation due to the average atomic number of the CdTe arm plus the core. Also, smaller variations of the image contrast can be seen in the other three arms of the tetrapod. In the image, none of the different tetrapod domains is in a precise zone axis. The core region is oriented close to the [11.1] zone axis of what is presumably the sphalerite $\mathrm{ZnTe}$ core, whereas the arms at the bottom and the top left are oriented close to the [12 $\overline{3} 0]$ and [2 $\overline{1} \overline{1} 3$ ] zone axes, respectively, of wurtzite CdTe. In this orientation, the third and fourth arms overlap and hence show a much brighter image intensity, close to saturation of the HAADF detector.

High-spatial-resolution EDS studies in STEM, on the other hand, can be used to directly address the chemical nature of the core of the tetrapod. ${ }^{39,41}$ In order to study the distribution of the chemical species in the tetrapods, spatially resolved EDS spectra were acquired while scanning an $\sim 0.2 \mathrm{~nm}$ electron probe along the nanostructure; $\sim 40 \mathrm{ZnTe} / \mathrm{CdTe}$ tetrapods were studied by EDS. Figure 2d shows, for instance, the HAADF image of a representative tetrapod along with the EDS spectra for $\mathrm{Cd}$ and $\mathrm{Zn}$ as a function of the electron probe position (the EDS spectrum for Te has been omitted for reasons of clarity of the figure). Here the EDS spectra were acquired along the yellow line and show the presence of $\mathrm{Zn}$ in the core region of the tetrapod. The shape of the EDS profile in the figure indicates that the $\mathrm{Zn}$-rich region is $\sim 5 \mathrm{~nm}$ wide. We caution the reader, however, that this evaluation overestimates the spatial distribution of the $\mathrm{Zn}$ region because of the broadening of the electron beam produced by the interaction with the relatively thick region of the outgoing CdTe arm. Indeed, the actual spatial distribution of the $\mathrm{Zn}$ in the core region deconvoluted from the beam broadening is $\sim 2.5 \mathrm{~nm}$ wide. ${ }^{57}$ Additionally, a few counts of Zn (slightly above the background level) can also be observed along the arm in the left part of the image and correspond also to a region of smaller intensity of the HAADF image contrast. This phenomenon could indicate the presence of an alloy containing $\mathrm{Zn}$ along the $\mathrm{CdTe}$ arm. It should be remarked that the $\mathrm{Zn}$ spectra obtained using the $\mathrm{K}$ lines and $\mathrm{L}$ lines have comparable features with respect to the spatial distribution. The presence of the $\mathrm{Zn}$ in the core of the tetrapods was found in $90 \%$ of the observed nanostructures, whereas in the remaining $10 \%$, the presence of X-rays characteristic of $\mathrm{Zn}$ was observed in neither the core nor the arms. This indicates that almost all

(56) Koo, B.; Korgel, B. A. Nano Lett. 2008, 8, 2490-2496.

(57) Goldstein, J. I.; Williams, D. B. In Principles of Analytical Electron Microscopy, 2nd ed.; Joy, J., Romig, A. D., Goldstein, J. I., Eds.; Plenum Press: New York, 1989; p 191. 
of the tetrapods in the sample formed via seeded growth and that a only small percentage of them nucleated homogeneously without the assistance of ZnTe seeds. Additionally, these results show that the seeded-growth approach reported here does not lead to complete interdiffusion of the chemical species of the core and the arms, in analogy with recently published results on $\mathrm{CdSe} / \mathrm{CdS}$-based nanorods and tetrapods grown from $\mathrm{CdSe}$ seeds ${ }^{40,41}$ in which the chemical nature of the core appeared to be preserved throughout the growth process.

The sample under study (as well as all of the tetrapod samples reported here) also contained some rods, dipods, and tripods. HAADF images of a dipod and a tripod are shown in Figure $2 \mathrm{e}, \mathrm{f}$. The fluctuations of the HAADF intensity in both images are probably due to small thickness variations in the structure that arise from the irregular profile of the arms. We carried out an EDS analysis on these structures also, and on most of them we found no presence of $\mathrm{Zn}$. This is remarkable and highlights the fact that most nanostructures with a lower degree of branching than tetrapods appear not to be formed by a seeded growth mechanism (i.e., via heterogeneous nucleation on a preformed seed) but rather result from homogeneous nucleation. Magnified views of the right and bottom arms in Figure $2 \mathrm{f}$ are shown in Figure $2 \mathrm{~g}$,h, where it is possible to distinguish the stacking sequences of planes along the arms. It is clear that the arms are highly defective, with random transitions from a sphalerite type of stacking sequence (ABCABC...) to a wurtzite type of stacking sequence (ABAB...). Additionally, one can distinguish regions in which there are sphalerite rotation twins (i.e., an inversion in the sphalerite stacking sequence from $\mathrm{ABCABC}$ to $\mathrm{CBACBA}$, such as in the central part of Figure $2 \mathrm{~g})$. Such a high density of defects was found on all of the nanocrystals (rods, bipods, tripods, and tetrapods) in which the arms were made of CdTe. This is somehow expected from $\mathrm{CdTe}$, as it has also been observed extensively in the growth of CdTe thin films. ${ }^{58,59}$

3.2. XRD Analysis and Simulation. XRD spectra of the seeds are reported in Figure S6 in the Supporting Information and confirm that all of the seeds were in the cubic sphalerite phase. On the basis of fits of the experimental patterns, the sizes of the CdSe, CdTe, and ZnTe seeds were found to be 1.9, 2.2, and $1.9 \mathrm{~nm}$, respectively. However, these fits tend to underestimate nanocrystal sizes, as the outermost planes in a nanocrystal are affected by surface reconstruction and can be amorphous, and additionally, planar defects contribute to decreasing the effective size of the monocrystalline scattering domains derived from such an analysis. Also, an error of $\sim 0.5 \mathrm{~nm}$ should be associated with this type of fit. Average CdSe, CdTe, and ZnTe seed sizes determined by TEM (see Figure S5 in the Supporting Information), on the other hand, were equal to 3,4 , and $3 \mathrm{~nm}$, respectively (these values were indeed those considered in the evaluation of the concentrations of seeds in the various syntheses). For CdSe and CdTe, these sizes were close to those reported in the literature on the basis of their optical absorption spectra. ${ }^{60}$ Figure 3 shows as red dotted lines the experimental powder diffraction patterns collected on three representative nanocrystal samples: (A) $\mathrm{CdSe} / \mathrm{CdTe}$, (B) ZnTe/CdTe, and (C) $\mathrm{ZnTe} / \mathrm{CdS}$ tetrapods as well as the corresponding simulated

(58) Yan, Y.; Al-Jassim, M. M.; Jones, K. M.; Wei, S. H.; Zhang, S. B. Appl. Phys. Lett. 2000, 77, 1461-1463.

(59) Yan, Y. F.; Al-Jassim, M. M.; Demuth, T. J. Appl. Phys. 2001, 90, 3952-3955.

(60) Yu, W. W.; Qu, L. H.; Guo, W. Z.; Peng, X. G. Chem. Mater. 2004, 16,560 .
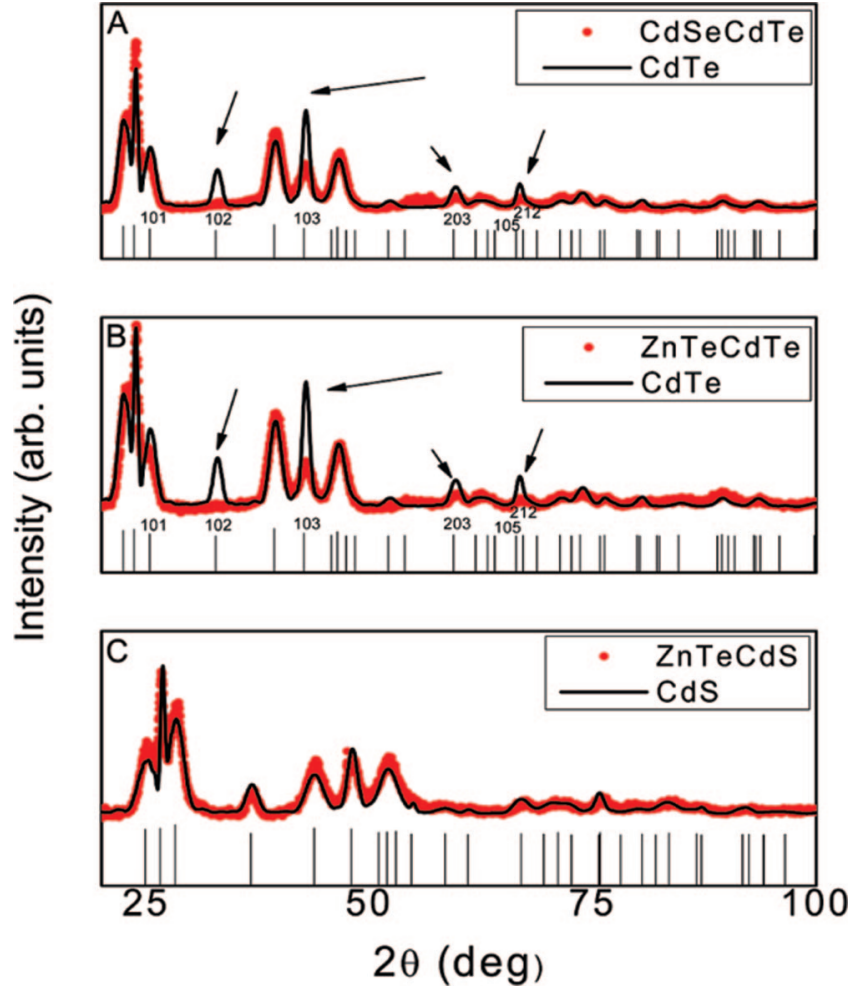

Figure 3. Experimental powder diffraction patterns (red dotted lines) and corresponding simulated patterns (black solid lines) for three representative nanocrystal samples: (A) CdSe/CdTe, (B) ZnTe/CdTe, and (C) ZnTe/CdS tetrapods. The structural models used for the XRD simulations were the following: (A, B) CdTe wurtzite rods of $60 \AA$ diameter and $150 \AA$ A length elongated along the 001 direction; (C) CdS wurtzite rods of $40 \AA$ diameter and $150 \AA$ A length elongated along the 001 direction.

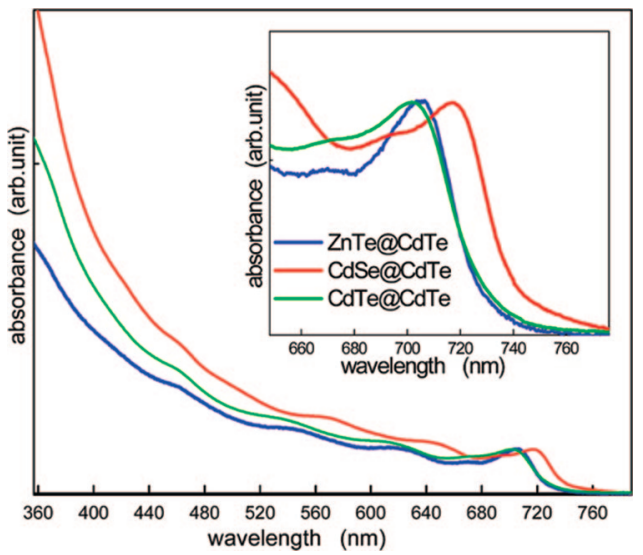

Figure 4. Linear absorption spectra of tetrapods with CdTe arms and either $\mathrm{ZnTe}$ cores (blue line), CdTe cores (green line), or CdSe cores (red line), normalized to the band-edge absorption. For these three samples, the first exciton peaks are at 706, 703, and $717 \mathrm{~nm}$ for the $\mathrm{ZnTe} / \mathrm{CdTe}, \mathrm{CdTe} / \mathrm{CdTe}$, and $\mathrm{CdSe} / \mathrm{CdTe}$ tetrapods, respectively. From low-resolution TEM measurements, the average diameter and length of the CdTe arms are 37 and $5 \mathrm{~nm}$ for the $\mathrm{ZnTe} / \mathrm{CdTe}$ tetrapods, 29 and $6 \mathrm{~nm}$ for the $\mathrm{CdTe} / \mathrm{CdTe}$ tetrapods, and 25 and $6 \mathrm{~nm}$ for the CdSe/CdTe tetrapods, respectively.

patterns as black solid lines. In all cases studied, the contribution to the diffraction from the central core (i.e., the original seed) was practically negligible, and therefore, the spectra could be modeled by considering as structural models wurtzite nanorods elongated along the 0001 direction whose lengths and diameters were the same as those of the arms of the tetrapods (as determined by TEM). We found excellent agreement between 

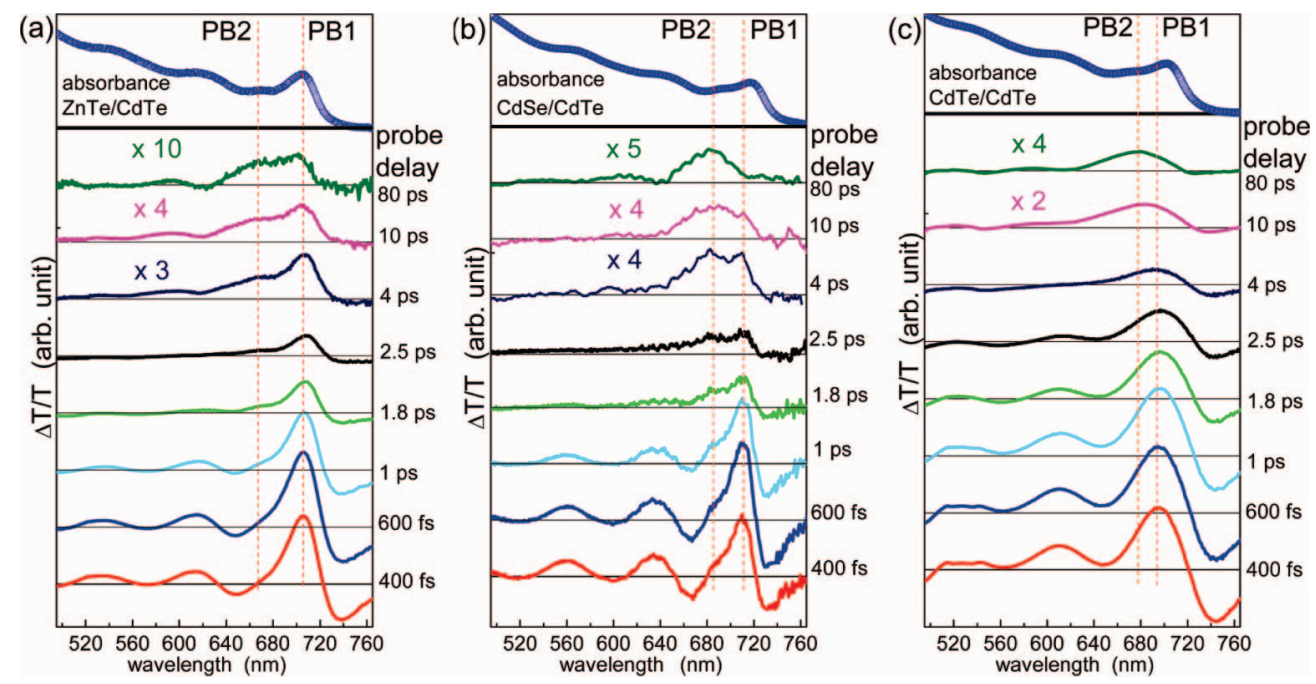

Figure 5. Time evolution of transient transmission spectra for (a) ZnTe/CdTe, (b) CdSe/CdTe, and (c) CdTe/CdTe tetrapods at different probe delays after pump excitation at $390 \mathrm{~nm}$.
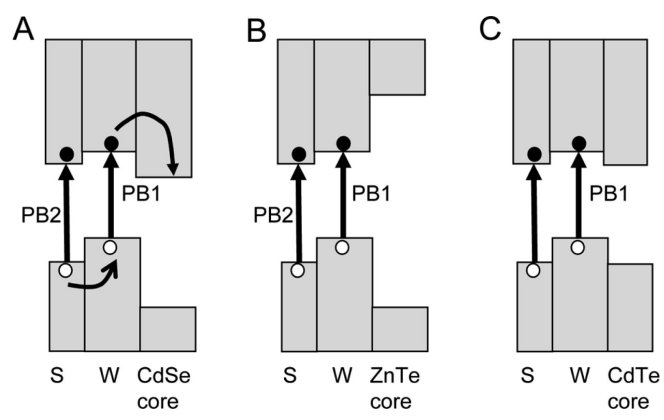

Figure 6. Sketches of the various level alignments for (A) CdSe/CdTe, (B) $\mathrm{ZnTe} / \mathrm{CdTe}$, and (C) CdTe/CdTe tetrapods. In the sketches, S and W are refer to sphalerite and wurtzite regions, respectively, in the CdTe arms.

experimental data and the simulated spectra for tetrapods with CdS arms (see, for instance, Figure 3C) but poorer agreement for tetrapods with CdTe arms, the latter due to the unsatisfactory description of the CdTe arms in terms of single-crystal domains in the pure wurtzite phase. In Figure 3A,B, the arrows indicate the most evident differences between the simulations and the data. Here a dampening in the intensities of the $10 l(l=1,2$, $3, \ldots)$ reflections was clearly observed and was interpreted as the fingerprint of a disordered wurtzite CdTe phase. Accordingly, the CdTe arms could be modeled better as composed of mixed regions of wurtzite and sphalerite phases (see Figure S7 in the Supporting Information for an additional simulation of the diffraction pattern). A similar dampening of the $10 l$ reflections was found, for instance, by Frey and Boysen ${ }^{61}$ for cobalt crystals grown in mixed hexagonal "hcp" and cubic "fcc" phases (the extent of this disorder was found to depend on the thermal history, purity, and grain size and the actual values of temperature and pressure at which the syntheses were carried out).

3.3. Pump-and-Probe Experiments. The absorption spectra of tetrapods with CdTe arms and either $\mathrm{ZnTe}, \mathrm{CdSe}$, or CdTe cores, normalized to the band-edge absorption, are reported in Figure 4. All of the spectra show similar shapes, regardless of the composition of the tetrapod core (whether $\mathrm{ZnTe}, \mathrm{CdSe}$, or $\mathrm{CdTe}$ ), indicating that the absorption is dominated by transitions

(61) Frey, F.; Boysen, H. Acta Crystallogr. 1981, A37, 819-826. arising from the CdTe arms. Slight differences in peak positions are associated with differences in the arm diameters of the various samples. In particular, the absorption spectrum of $\mathrm{CdSe} /$ CdTe tetrapods does not indicate any evidence of spatially indirect transitions associated with type-II band alignment. However, in view of the fact that the contribution of the CdSe core is almost negligible in terms of absorbance, any absorption band due to spatially indirect transition would be extremely weak and hence difficult to detect. On the other hand, type-II carrier recombination, with the associated emission in the infrared region, was observed for such tetrapods (see below).

The chirp-free differential transmission $(\Delta T / T)$ spectra for tetrapods with CdTe arms and different cores at various probe delay times after pumping are reported in Figure $5 \mathrm{a}-\mathrm{c}$ for $\mathrm{ZnTe}$, $\mathrm{CdSe}$, and CdTe cores, respectively. All of the spectra show photobleaching $(\mathrm{PB})$ and a superimposed photoinduced absorption (PA); in particular, a strong PA is very evident at $730 \mathrm{~nm}$ and probably underneath the whole spectrum. The three samples have similar behaviors. The initial $\Delta T / T$ spectra are due to a superposition of bleaching (due to state filling) and PA (possibly originating from Coulomb effects ${ }^{62}$ or trap states ${ }^{63,64}$ involving hot electron-hole pairs). Both the lower-wavelength bleaching features and the PA disappear in a few picoseconds, leaving the bleaching structure above $600 \mathrm{~nm}$.

For all of the samples, the bleaching at the first exciton transition (PB1) has buildup dynamics, reaching a maximum at $\sim 600 \mathrm{fs}$, and possibly can be assigned to thermalization. A second bleaching feature (PB2) at shorter wavelength appears at a slightly longer time. The buildup of PB2 is quite evident in tetrapods having $\mathrm{CdSe}$ and $\mathrm{ZnTe}$ cores, whereas in those with CdTe cores, PB1 and PB2 are less resolved because of a smaller difference in energy, and only one bleaching peak is evident. In the tetrapods with ZnTe cores, PB1 and PB2 are very evident at probe delays of up to $80 \mathrm{ps}$, whereas in the sample with $\mathrm{CdSe}$ cores, $\mathrm{PB} 1$ decays faster than PB2: it is strongly reduced after 10 ps and disappears after 80 ps. The CdTe/CdTe transient spectra show a blue shift of the broad bleaching peak, suggesting

(62) Klimov, V. I. J. Phys. Chem. B 2000, 104, 6112-6123.

(63) Cretì, A.; Anni, M.; Rossi, M. Z.; Lanzani, G.; Leo, G.; Della Sala, F.; Manna, L.; Lomascolo, M. Phys. Rev. B 2005, 72, 125346.

(64) Cretì, A.; Zavelani-Rossi, M.; Lanzani, G.; Anni, M.; Manna, L.; Lomascolo, M. Phys. Rev. B 2006, 73, 165410. 


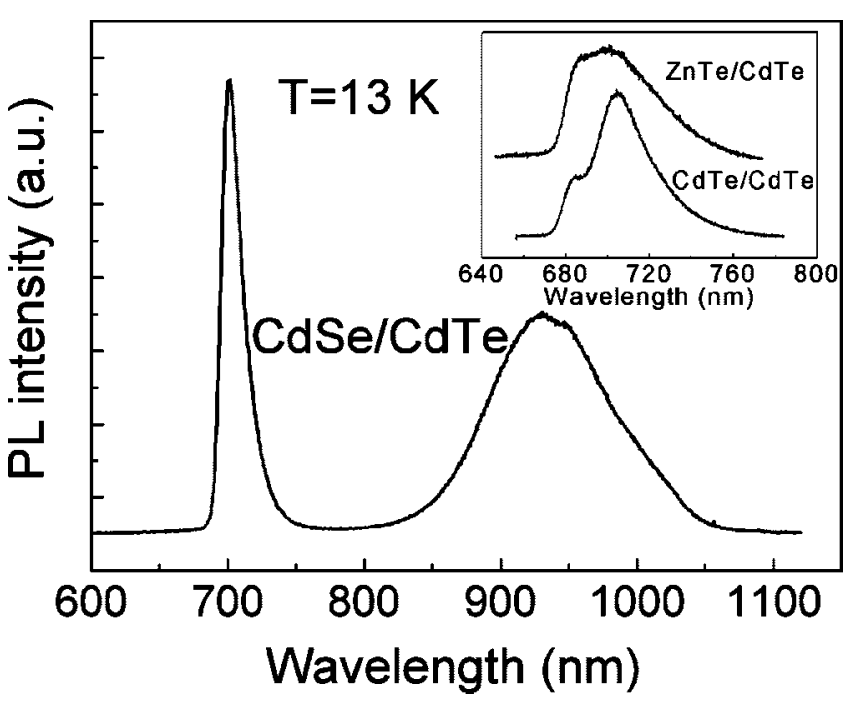

Figure 7. $\mathrm{PL}$ spectrum of $\mathrm{CdSe} / \mathrm{CdTe}$ tetrapods recorded at $13 \mathrm{~K}$. Inset: $\mathrm{PL}$ emission from $\mathrm{ZnTe} / \mathrm{CdTe}$ and $\mathrm{CdTe} / \mathrm{CdTe}$ tetrapods, also recorded at $13 \mathrm{~K}$.

that $\mathrm{PB} 2$ is retained while $\mathrm{PB} 1$ is reduced, an evolution strictly resembling that of the $\mathrm{CdSe} / \mathrm{CdTe}$ tetrapods.

We conjecture that PB1 and PB2 stem from different regions in the nanocrystal. As a matter of fact, HRTEM analysis reveals crystallographic disorder along the CdTe arms. Sphalerite regions embedded in the wurtzite structure would localize electrons, which are mainly responsible for $\mathrm{PB}$, due to a typeII alignment ${ }^{65-67}$ (see Figure 6). It should be noted that the estimated length scale of the disorder is $\sim 2-4 \mathrm{~nm}$, and the strong quantum confinement expected in the localizing regions is consistent with the higher energy of the PB2 transition. The $\mathrm{PB}$ evolution in tetrapods may be associated with the different types of level alignment at the core-arm interface. In $\mathrm{CdSe} /$ CdTe and CdTe/CdTe tetrapods, because of type-II alignment ${ }^{65}$ (the CdTe core has a sphalerite structure), electrons can be transferred from the arms to the core. This would result in the suppression of PB1. In ZnTe/CdTe tetrapods, both carriers are confined in the arms (see Figure 6).

On the basis of the pump-probe results, charge separation is therefore evident in these nanocrystals. Along the CdTe arms in all of these tetrapods, electrons can be localized in nanometer regions while holes are delocalized in more extended areas. In type-II core/arm tetrapods, an additional separation of charge between the arms and the core occurs, giving rise to electron localization in the core region. The effect is more evident in the $\mathrm{CdSe} / \mathrm{CdTe}$ tetrapods and was confirmed by PL experiments.

(65) Landolt-Börnstein, New Series, Group III; Springer: Berlin, 1982; Vol. $17 \mathrm{~b}$.

(66) Klimov, V. I.; McBranch, D. W.; Leatherdale, C. A.; Bawendi, M. G. Phys. Rev. B 1999, 60, 13740-13749.

(67) Yeh, C. Y.; Lu, Z. W.; Froyen, S.; Zunger, A. Phys. Rev. B 1992, 46, 10086-10097.
Low-temperature PL spectra recorded for the three tetrapod samples (Figure 7) show a PL emission centered at $\sim 710 \mathrm{~nm}$ for all of the samples, whereas only the CdSe/CdTe sample is characterized by a second PL emission peak in the infrared region (at $\sim 950 \mathrm{~nm}$ ). The different band alignments for the three structures, depicted in Figure 6, suggest that the emission at $\sim 710 \mathrm{~nm}$ is related to a type-I radiative recombination in the CdTe arms, whereas the emission in the infrared region of the $\mathrm{CdSe} / \mathrm{CdTe}$ sample should correspond to the type-II transition arising from the recombination of electrons in the CdSe core and holes in the CdTe arms (see Figure 7).

\section{Conclusions}

We have developed a general protocol for the synthesis of tetrapod-shaped nanocrystals of II-VI semiconductors based on a seeded-growth approach, which allowed us to vary independently the compositions of the core and the arms of the tetrapods. The yield in uniform tetrapods was much higher than when no seeds were coinjected. In tetrapods with CdTe arms, the more defective crystal structure of the arms, which is due to the presence of both sphalerite and wurtzite domains, was assessed by a combination of structural and optical techniques. We believe that the approach described in this report can be extended to include the preparation of tetrapods of other combinations of materials (for instance, mixed II-VI/III-V tetrapods), and work in this direction is currently in progress in our groups. Furthermore, this new generation of more uniform and composition-tunable tetrapods should find direct application in hybrid polymer-nanocrystal photovoltaic devices.

Acknowledgment. This work was supported by the European SA-NANO Project (Contract STRP 013698) and by the Italian Ministry of Research (under Contracts RBLA03ER38 and FARDM18604). We acknowledge Professor Giuseppe Gigli for many inspiring discussions and Giuseppe Chita and Roberto Lassandro for assistance with the XRD measurements.

Supporting Information Available: TEM images comparing various nanocrystals synthesized with and without coinjection of seeds and using sphalerite seeds; TEM figures showing results of representative syntheses carried out at surfactant ratios and temperatures different from those reported in the main text; TEM images of seeds used in the syntheses of the heterostructures; experimental and simulated XRD spectra of a sample of $\mathrm{CdSe} /$ CdTe tetrapods; experimental diffraction patterns and related fits for the various seeds used in the syntheses; optical absorption spectra of the various seeds and of all of the corresponding tetrapods; a wide-field TEM image of a sample of $\mathrm{ZnTe} / \mathrm{CdTe}$ tetrapods; and complete ref 40. This material is available free of charge via the Internet at http://pubs.acs.org.

JA807874E 